\title{
SCMR level II/independent practitioner training guidelines for cardiovascular magnetic resonance: integration of a virtual training environment
}

\author{
Amit R. Patel ${ }^{1^{*}} \mathbb{D}$, Sebastian Kelle ${ }^{2}$, Marianna Fontana ${ }^{3}$, Ron Jacob ${ }^{4}$, Jadranka Stojanovska ${ }^{5}$, Jeremy Collins ${ }^{6}$, \\ Hena N. Patel ${ }^{1}$, Marco Francone ${ }^{7,8}$, Yuchi Han ${ }^{9}$, W. Patricia Bandettini ${ }^{10}$, Chiara Bucciarelli-Ducci ${ }^{11}$, \\ Subha Raman ${ }^{12}$ and Gaby Weissman ${ }^{13}$
}

Cardiovascular magnetic resonance (CMR) is an important non-invasive imaging modality used for the evaluation of patients with known or suspected heart disease. Despite its clinical importance, CMR is currently not widely available, in part, because of a scarcity of welltrained physicians to perform and interpret the exam. Moreover, current 2018 Society for Cardiovascular Magnetic Resonance (SCMR) training guidelines [1] and also training guidelines from other societies $[2,3]$ require a significant amount of in-person hands-on experience making training inaccessible for many individuals. This limits the availability of physicians appropriately trained to perform and interpret CMR exams. The purpose of this statement is to provide guidance for implementing a high-quality virtual CMR training program to complement in-person training options.

By leveraging advancements in video communication platforms, Level II or Independent Practitioner competence in CMR is feasible through the use of a virtual training program that substitutes a portion of the required in-person training with the use of two-way interactive video communication equipment, provided

\footnotetext{
*Correspondence: amitpatel@uchicago.edu

${ }^{1}$ Department of Medicine and Radiology, University of Chicago, Chicago, IL, USA

Full list of author information is available at the end of the article
}

there is active participation between the trainee and the mentor. This two-way communication can be utilized to supplement all elements of a training program, including didactics, protocol selection, image acquisition, and image analysis. A description of the Level II or independent practitioner training requirements is detailed in the 2018 SCMR training guidelines [1].

In order to ensure quality training of CMR is accessible to qualified physicians interested in performing CMR, the SCMR will consider verifying Level II CMR training for those physicians who have demonstrated competence in CMR and have received CMR training as determined by the requirements set forth by SCMR while substituting the in-person, hands-on experience with a high-quality, two-way, video-communication experience. The goal of these recommendations is to maintain high quality CMR level II training and to keep the standards that are currently established for the in-person training environment, while providing guidance for the use of innovative educational approaches, such as the use of virtual training and available digital tools. Acknowledging the importance of the one-on-one interaction between the mentor and the trainee, the interactive sessions of the virtual training (such as the image interpretation and image acquisition) should be limited to a small group of trainees per mentor; whereas didactic sessions could have a larger number of trainees or be pre-recorded.

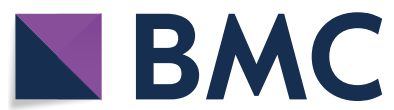

(c) The Author(s) 2022, corrected publication 2022. Open Access This article is licensed under a Creative Commons Attribution 4.0 International License, which permits use, sharing, adaptation, distribution and reproduction in any medium or format, as long as you give appropriate credit to the original author(s) and the source, provide a link to the Creative Commons licence, and indicate if changes were made. The images or other third party material in this article are included in the article's Creative Commons licence, unless indicated otherwise in a credit line to the material. If material is not included in the article's Creative Commons licence and your intended use is not permitted by statutory regulation or exceeds the permitted use, you will need to obtain permission directly from the copyright holder. To view a copy of this licence, visit http://creativecommons.org/licenses/by/4.0/. The Creative Commons Public Domain Dedication waiver (http://creativecommons.org/publicdomain/zero/1.0/) applies to the data made available in this article, unless otherwise stated in a credit line to the data. 
The image analysis and initial interpretation of a CMR exam remains an essential part of the competency assessment. The percentage of cases that may be replaced by a virtual experience can vary depending on (1) the program logistics and its curriculum, (2) the time spent by the program faculty in mentoring trainees, and (3) the competency of the individual trainee as evaluated by the CMR mentor. Importantly, a final assessment of the trainee's competency may still require an in-person training component allowing the mentor to observe the trainee's ability to acquire and interpret a CMR examination.

A high-quality virtual experience should include, but not be limited to, the following stipulations:

- The virtual training program should be fully compliant with the current SCMR training guidelines [1] and ensure the trainee achieves adequate competency to independently perform and interpret CMR studies

- Virtual training program should be led by a Level-IIItrained, Advanced Practitioner, or equivalent CMR expert

- Training centers must have a successful track record of on-site training as a pre-requisite for providing virtual SCMR Level II CMR training courses

- Didactic lectures can be provided by faculty selected by the training program. Alternatively, pre-recorded lecture series similar to those provided by the SCMR can be utilized

- In order to allow for direct observation and interaction with the scans as required by the SCMR training guidelines, the trainee must be able to visualize the control console during the CMR scan

- The trainee should have the opportunity to either provide input with sequence selection and modification/troubleshooting based on the clinical question and patient characteristics or have dedicated sessions focused on image planning and trouble-shooting

- The virtual training program should include full interpretation of CMR cases presented in a complete study format with review and feedback from the mentor. These cases can be previously acquired at the training center or obtained through the use of registries such as the SCMR registry to ensure that the full breadth of disease states are encountered by the trainee

- The virtual training program must incorporate safety considerations related to performing CMR. Depending on a trainee's experience, some components of CMR training such as (but not limited to) stress testing and supervision of individuals with cardiac implantable electronic devices may require an in-person experience
- Video communication equipment should meet local standards for analyzing medical images

- Patient privacy issues should meet local policies and regulations

- The specific remote training plan can be reviewed by SCMR for input if desired by the training program

- The trainee and training program are encouraged to provide feedback to the SCMR about the perceived successes and failures of the virtual training program.

The final determination of a trainee's competency to independently perform and interpret CMR studies is up to the program director of a training program. This document provides guidance to those developing a virtual CMR training program to complement in-person training. SCMR would be willing to verify that a virtual CMR training program meets the above standards prior to the initiation of the program. While further documents will need to address the future state of CMR training, it is expected that future CMR training will include both a combination of in-person training as well as incorporation of the virtual tools that are available.

Virtual training in CMR provides increased educational flexibility that is currently necessary but its effectiveness will need to be periodically reassessed. The feedback of mentors and trainees will be highly valuable to address future needs in education. The experiences of these virtual training courses will potentially strengthen the ability to train physicians in locations where CMR might not be readily available, and therefore spread its appropriate utilization. Further, adapting a framework that combines virtual and more traditional in-person education may better fit the needs and expectations of newer generations of physicians. We anticipate that future training models will utilize all the tools and techniques available to provide high quality training to a diverse and broad group of physicians allowing for the utilization of CMR in a greater number of locations. The use of new technologies for CMR training will continue to supplement high quality in-person education and lead to a highly skilled and well-trained CMR practitioner workforce.

\section{Authors' contributions}

All authors participated in multiple meetings and communications to determine the contents of the document. All authors helped write the manuscript. All authors read and approved the final manuscript.

\section{Declarations}

\section{Competing interests}

The authors do not have any relevant conflicts of interest directly related to the topic of this manuscript. ARP has received unrelated research funding or support from General Electric, Philips Healthcare, CircleCVI, Arterys, and Neosoft. CBD is the chief executive officer for the Society of Cardiovascular 
Magnetic Resonance and has received speaking fees from Circle Cardiovascular Imaging, Bayer, and Siemens Healthineers.

\section{Author details}

'Department of Medicine and Radiology, University of Chicago, Chicago, IL, USA. ${ }^{2}$ Department of Internal Medicine/Cardiology, German Heart Center Berlin, Berlin, Germany. ${ }^{3}$ Division of Medicine, National Amyloidosis Centre, University College London, London, UK. ${ }^{4}$ The Heart and Vascular Institute, Lancaster General Health/Penn Medicine, Lancaster, PA, USA. ${ }^{5}$ New York University, New York City, NY, USA. ${ }^{6}$ Department of Radiology, Mayo Clinic, Rochester, MN, USA. ${ }^{7}$ Department of Biomedical Sciences, Humanitas University, Pieve Emanuele, Milan, Italy. ${ }^{8}$ Humanitas Research Hospital IRCCS, Rozzano, Milan, Italy. ${ }^{9}$ Cardiovascular Division, Department of Medicine, Perelman School of Medicine, University of Pennsylvania, Philadelphia, PA, USA. ${ }^{10}$ Division of Intramural Research, Cardiology Branch, National Heart, Lung, and Blood Institute, National Institutes of Health, Bethesda, MD, USA. ${ }^{11}$ Royal Brompton and Harefield Hospitals, Guys' and St Thomas NHS Hospitals and School of Biomedical Engineering \& Imaging Sciences, King's College London, London, UK. ${ }^{12}$ Krannert Institute of Cardiology, Indiana University School of Medicine, Indianapolis, IN, USA. ${ }^{13}$ Department of Cardiology Medstar Heart and Vascular Institute, Georgetown University, Washington, DC, USA.

Received: 24 November 2021 Accepted: 30 November 2021

Published online: 27 December 2021

\section{References}

1. Kim RJ, Simonetti OP, Westwood M, et al. Guidelines for training in cardiovascular magnetic resonance (CMR). J Cardiovasc Magn Reson. 2018;20:57

2. Kramer CM, Hudley WG, Kwong RY, Martinez SV, Ward RP. COCATS 4 task force 8: training in cardiovascular magnetic resonance imaging. J Am Coll Cardiol. 2015;65(17):1822-31.

3. ACR-NASCI-SPR practice parameter for the performance and interpretation of cardiac magnetic resonance imaging (MRI). American College of Radiology Revised 2016 (Resolution 5).

\section{Publisher's Note}

Springer Nature remains neutral with regard to jurisdictional claims in published maps and institutional affiliations.

Ready to submit your research? Choose BMC and benefit from:

- fast, convenient online submission

- thorough peer review by experienced researchers in your field

- rapid publication on acceptance

- support for research data, including large and complex data types

- gold Open Access which fosters wider collaboration and increased citations

- maximum visibility for your research: over $100 \mathrm{M}$ website views per year

At BMC, research is always in progress.

Learn more biomedcentral.com/submissions 